\title{
How can multimodality be used to design usable interfaces in IPS $^{2}$ for older employees?
}

\author{
Ulrike Schmuntzsch ${ }^{\mathrm{a}, *}$, Christine Sturm ${ }^{\mathrm{a}}$ and Matthias Roetting ${ }^{\mathrm{a}}$ \\ ${ }^{a}$ Chair of Human-Machine Systems, Department of Psychology and Ergonomics, Technische Universität Berlin, \\ Franklinstr. 28-29, 10587 Berlin, Germany
}

\begin{abstract}
Main objective of the recently started collaborative research project SFB/TR29 B4 is to provide cross-generational assistance to human operators working in industrial product-service systems (IPS ${ }^{2}$. By combining the ideas of actionspecificity and multimodality a more immediate and purposeful reaction towards warnings is assumed. For this purpose, an action-specific warning system in form of a glove has been developed. It provides either visual, auditory or haptic feedback or a combination of all modalities. In a first study this prototype was compared with a conventional warning system, where multimodal devices were directly mounted to the machine. The test scenario used in this study is the "changing of a spindle' on a replica of a micro milling machine. It was conducted with 42 participants. The experimental design is a within-design in terms of the two warning systems and a between-design concerning the three modalities and its combinations. Results of the two-way ANOVA with repeated measures on both factors 'system' and 'modality' show a significant main effect for the 'modality' and a significant interaction effect between both factors. Especially the haptic warnings on the action-specific glove were described as "that kind of warning, which evokes the fastest response".
\end{abstract}

Keywords: action-specific warning, multimodality, cross-generational interface design, industrial product-service systems

\section{Introduction}

How can multimodality be used to design usable interfaces in IPS ${ }^{2}$ for older employees? Is this question and the search for potential solutions of any importance to human-machine interaction? Yes, it is.

Modern industrialized countries are nowadays confronted with various challenges. Two of the most pressing ones are the ongoing demographic change and the growing global competition. As to the demographic change an increasing average age of the workforce is expected. This leads to a lack of young employees and with that to a shortage of workers skilled in recent technologies. Consequently, companies in industrialized countries are more and more forced to create new concepts and technologies which help older employees to master their work as long as possible. Moreover, these companies have to face an increasing global competition with emerging nations like India or Brazil. As a result, especially in the field of industrial production, growing specialization and complexity within supply chains has already begun. Technical equipment and procedures become increasingly multifaceted and require advanced expert knowledge. These challenges are glaringly apparent in the area of Industrial Product-Service Systems $\left(\right.$ IPS $\left.^{2}\right)$. In IPS ${ }^{2}$ the design, development, provision and utilization of products and services are conducted, integrated and mutually dependent [4]. Thus, IPS $^{2}$ can be regarded as socio-technical systems involving mechanical components and human factors. The latter one comprises a variety of congenital and acquired human-specific abilities and skills such as the physical and mental constitution [13]. The wellfunctioning interplay between human operator and machine is one key factor for the safety and productivity of the whole socio-technical system. As mentioned before, the dynamic and complex structure of

Corresponding author. E-mail: ulrike.schmuntzsch@mms.tu-berlin.de. 
IPS $^{2}$ puts high demands on the human operator such as flexible and immediate actions as well as specialized system knowledge. Older employees may be reluctant to face up to all these challenges. Therefore, new concepts and technologies are urgently required.

Main objective of the recently started second phase of the collaborative research project SFB/TR29 B4 is to provide user assistance in case of operating errors and system malfunctions. By assisting users the reliability and performance of the whole humanmachine system can be optimized. For that reason, it is intended to develop multimodal action-specific warnings and instructions for human operators working in IPS ${ }^{2}$. These error situations are fundamental to usability on two fronts: danger to man, machine, or environment and knowledge enhancement. The former focuses on a potential harm and at the risk that the targeted objective will not be achieved. The latter describes the option to support the user in satisfactorily accomplishing the tasks and finally understanding the system better. As a consequence, warnings can be considered as an integral part of an overall design strategy.

The following article describes an approach to design a multimodal user interface that provides actionspecific warnings for human operators in IPS ${ }^{2}$. This includes a description of the developed prototype and the first experiment with its main results. Chapter two starts with a brief introduction to the theoretical background concerning human error, multimodality and main design aspects of warnings as well as the most relevant facts of human perception and special challenges in terms of aging. Afterwards, in chapter three, our prototype, the procedure of the conducted study and its first findings will be presented. The article finishes in chapter four with a discussion of the experimental results and a brief outline of future considerations.

\section{Theoretical background}

\subsection{Human error categorization}

As previously mentioned, error situations can have many different causes, e.g. system malfunctions and/ or human errors. Regarding the latter one it has to be distinguished between latent errors and active errors. Latent errors are mostly organizational deficiencies, which often remain unnoticed for a long time. Active errors are directly obvious and strongly related to immediate consequences. These kinds of errors are characterized as triggering events and are called operating errors [9].

Rasmussen [8] graded human operations and thereby occurring errors into three different levels depending on the resources applied. These were named skill-based, rule-based and knowledge-based operations and errors. Skill-based operations are executed automatically without paying conscious attention. Associated errors are known as slips or lapses. When pointed out, the user becomes aware of them and as a matter of fact knows how to perform properly. Rulebased errors are caused by wrong or incomplete interaction patterns. The execution itself is accurate, but inappropriate in this particular case. Users will behave correctly even in this context, once their inappropriate action selection is pointed out to them. Solely in the last case of knowledge-based errors, a lack of knowledge on how to master the task is causative for the error. At this point it is not only necessary to provide action-specific warnings, but also to give further instructions. Both aspects are part of the $\mathrm{SFB} / \mathrm{TR} 29 \mathrm{~B} 4$ project, but this article focuses exclusively on the action-specific warnings.

\subsection{Multimodality and warnings}

Warnings can be considered as an essential part of an overall design strategy. Regarding the aspect of multimodality various studies show the many advantages of user interfaces, if warnings were applied properly. According to Oviatt [6], multimodal interfaces are characterized by two or more input and output modalities, which are combined in a coordinated manner. The term "multimodal" includes the type and number of appealed human senses as well as the possibility of parallel processing [1]. Current interfaces mainly focus on auditory, visual and tactile senses [3]. With an appropriate design, multimodal interfaces offer several advantages, such as adapting to the individual abilities and skills of users as well as to changing environmental conditions. Furthermore, results of different studies indicate superiority of multimodal over unimodal interfaces with respect to error prevention and correction [7].

When it comes to warnings, it is of special importance to make optimal use of multimodality. If warnings do not correspondent to the way users usually perform their task, it may result in difficulties in understanding warnings and instructions. That reveals an information gap between error message and correct task performance by the user [5]. One can say 
that warnings and instructions are a sensitive subject. Users may feel either insecure, confused or even incompetent, after making mistakes inadvertently or out of lack of knowledge. Some users could even be scared when e.g. hearing a penetrating tone. So, in order to help users to correct a problem, constructive warnings should be formulated positively and appear in an appropriate way [11].

In summary, warnings are on the one hand important to help shape the users understanding of the system. On the other hand warnings are supposed to fit to the user's mental model of the system in order to help him or her accomplishing the task as usual.

\subsection{Psychological and physiological aspects of aging}

On a cognitively-oriented level, various steps are essential for information processing. In simplified terms, the detection of relevant stimuli via senses can be considered as a first step, mainly affected by bottom-up processes. The quality of this depends on many different aspects such as the perceptible signal strength and the surrounding conditions. Furthermore, top-down processes such as quality of the cognitive model and relevant process knowledge are important as well. On the basis of signal identification an alignment with already existing knowledge in the long-term memory can be done (top-down processes). Hereafter follow the decision-making process, the action selection and action implementation [2]. According to the complete process one thing is certain: the detection and identification of relevant signals create a major prerequisite for an immediate and appropriate response when a warning is given.

A number of perceptual and cognitive processes are affected by increasing age (see Table 1). Thus, to ensure a cross-generational design of multimodal interfaces and particularly of warnings, various psychological and physiological aspects of aging have to be well considered. Those aspects have an influence on perception, motor skills and cognitive processes [12].

Table 1

Age-related perceptual and cognitive effects according to Weißmantel \& Biermann [12]

\begin{tabular}{|l|l|l|}
\hline Function & Category & Age-related effects \\
\hline Perception & Visual & $\begin{array}{l}\text { Increased sensitivity to glare; decreased depth perception, visual acuity and accommodation; } \\
\text { delayed dark adaptation and sharpness perception; a narrowing of the visual field }\end{array}$ \\
\cline { 2 - 3 } & Auditory & $\begin{array}{l}\text { Presbycusis; susceptibility to background noise; side-to-side different loss in dichotic } \\
\text { discrimination }\end{array}$ \\
\cline { 2 - 3 } & Haptic & Decreased sensitivity of touch and skin sensitivity \\
\hline Cognition & $\begin{array}{l}\text { Information } \\
\text { Processing }\end{array}$ & Decreased amount of simultaneously processed information and increased processing time \\
\cline { 2 - 3 } & Memory & Slower learning of new facts; reduced scope of actively and passively memorizable information \\
\cline { 2 - 3 } & Reaction & Increased reaction time and decreased reaction rate, specially in complex tasks \\
\cline { 2 - 3 } & Coordination & $\begin{array}{l}\text { Limited ability to coordinate action execution, extended sensitivity to interference with sensory } \\
\text { overload, limited capability to resume actions }\end{array}$ \\
\hline
\end{tabular}

Basically, the consideration of these effects in interface design is not only important for older users, but generally improves perceptibility and processing of information across all user groups [12]. For that reason these aspects are considered in the design of multimodal information and warnings as part of the SFB / TR 29 B4 project.

\section{Method}

\subsection{Action-specific warning system - concept and prototype}

Toady's conventional warning systems are usually designed multimodally, but being located directly at the machine the action-specific part is missing. Thus, emerging warnings can cause confusion for 
users precisely because of lacking a direct concern to the user's actions. As a result, useful response time is probably passing by.

Relating to the introductorily presented demographic and economic challenges in the field of IPS ${ }^{2}$ as well as to the theoretical background of multimodal warnings and human information processing, to design a warning system, which is closer related to the human operator's specific actions, seems to be glaringly important. According to that concept of action-specific warnings not only a simple warning is provided to the user, but within more detailed information about the action-specific reasons and the error's location. Combining the ideas of actionspecificity and multimodality a cross-generational support for human operators working with multifaceted and complex technical equipment will be realized. Consequently, a more immediate and purposeful reaction towards warnings can be assumed.

The prototypic realization of the concept of action-specific warnings is a glove, to which multimodal warning devices - 6 LED for visual feedback, 2 vibrating elements for haptic and one speaker for auditory - were attached as seen in Figure 1.

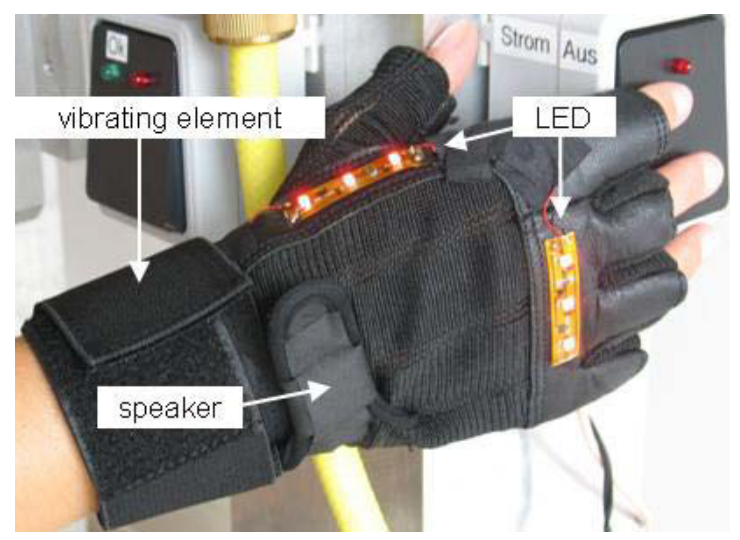

Fig. 1 Glove (action-specific warning)

\subsection{Spindle change scenario}

In order to test the developed prototype a experiment was conducted. In this study, two warning systems were compared with each other: a conventional system and the new action-specific system. The test scenario used in this study is the "changing of a spindle' on a milling machine used in micro production, where a broken spindle is to be replaced by a new one. For the experiment, the most likely, respectively the most dangerous errors while working on the machine were identified by experts in the field of milling. They came up with six errors. Each of these errors will lead to a warning. All warnings were induced by the Wizard-of-Oz-method.

For this study a replica of a micro milling machine was build as seen in Figure 2. On a table to the right of the replica a laptop displaying the warning messages was positioned. At the conventional system the warning devices were mounted directly to the micro milling machine. Implementations of visual, auditory and haptic warnings were the following: visual - 6 LED, auditory - a little speaker, haptic -2 vibrating elements. In order to realize the Wizard-of-Oz-technique warnings were induced by the experimenter using an Arduino micro controller (ATMega32). Warnings appeared 5 times each and in combinations of modalities the blinking, beeping and vibrating occurred simultaneously.

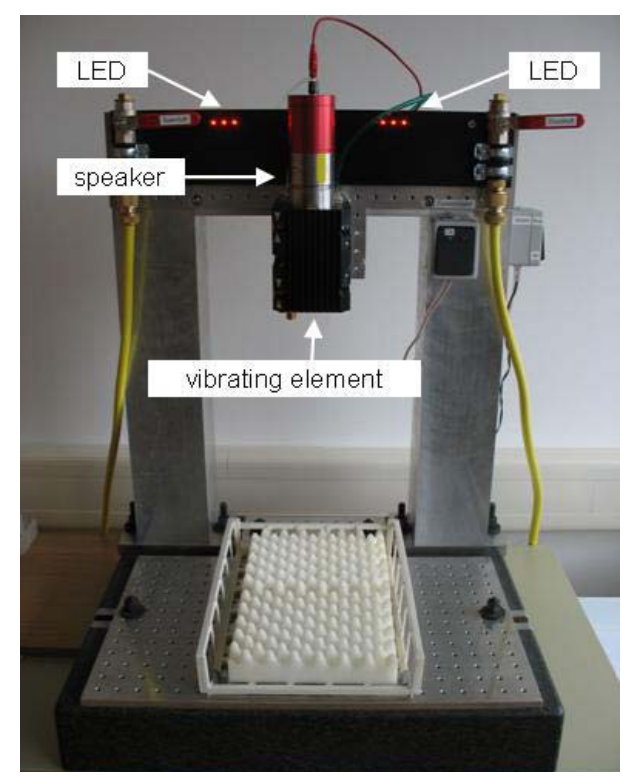

Fig. 2 Replica of a micro milling machine equipped with a conventional warning system

In this study a conventional warning system and an action-specific system were used. Note that in both systems error and warning correlated. However, the glove (as action-specific warning system) additionally contained information about the error's location. Thus, both systems differ in means of the action-specific information, which only the glove provides.

While working on the spindle change task, participants were recorded with two video cameras, which were installed up above to the left and right of the micro-milling machine. The video cameras were 
also used to realize the Wizard-of-Oz-technique as participants could be observed via life video transmission. What participants said during the debriefing session was recorded with a conventional dictating machine. Their ratings of both warning systems were photographed with a digital camera.

\subsection{Experimental design}

The seven warning modalities tested in this study were visual, auditory, haptic and their combinations visual-auditory, visual-haptic, auditory-haptic as well as visual-auditory-haptic. Only one modality, respectively a combination of modalities was presented as warning to each participant while working on the machine (between-design). However, after completing the task, all other modalities were dem- onstrated as well. This was important since participants were supposed to rate all modalities in the debriefing session, which then again led to a within design in the analysis.

Each participant came in contact with both warning systems and was always exposed to the same three warnings per system. During the first part of the spindle change task (removal of the old spindle) participants were warned by either the glove or the conventional warning system and during the second part (installing the new spindle) warnings originated from the respectively other system (within-design). To counterbalance order effects the systems were equally used as first, respectively second warning system (see Table 2).

Table 2

Number of participants in each warning modality

\begin{tabular}{|l|l|l|l|}
\hline Presentation (within) & Conventional system first & Action-specific system first & Sum \\
Test modality (between) & & 3 & 6 \\
\hline Visual & 3 & 3 & 3 \\
\hline Auditory & 3 & 3 & 6 \\
\hline Haptic & 3 & 3 & 6 \\
\hline Visual-auditory & 3 & 3 & 6 \\
\hline Visual-haptic & 3 & 3 & 6 \\
\hline Auditory-haptic & 3 & 21 & 6 \\
\hline Visual-auditory-haptic & 3 & 62 \\
\hline Sum & 21 & 6 \\
\hline
\end{tabular}

\subsection{Procedure}

The experiment lasted about 30 minutes and can broadly be divided in two parts: firstly, exposing the subjects to the warnings as they changed the spindle (test scenario) and secondly, questioning subjects about their experiences with those warnings (questioning part).

\subsubsection{Test scenario}

After a brief introduction to the purpose of the study, participants were asked to work on the spindle change task with help of an instruction manual. The manual contained detailed step by step instructions on how to change the spindle. The test scenario itself consisted of two main parts: removing the bro- ken spindle and installing a new spindle. Participants were told that during the task, as part of the study, several warnings would occur. In case of a warning, they were asked to look at the laptop display, where a detailed error message was shown, as well as a hint on how to solve the problem. After solving the problem, subjects would go on with the task according to the manual.

\subsubsection{Questioning part}

The questioning part started off by asking participants to rate the appropriateness of all warnings. For this evaluation a rating matrix (see Table 3) was prepared as well as little cards for each of the seven warning modalities. The cards had to be placed on the rating matrix by the participant. At first, partici- 
pants were asked to rate on a verbal scale how suitable, convenient respectively appropriate they think each warning modality was: very appropriate (sehr geeignet), fairly appropriate (ziemlich geeignet), moderately appropriate (mittelmäßig geeignet), hardly appropriate (wenig geeignet), not appropriate at all (gar nicht geeignet) according to Rohrmann [10]. In a second step, participants should indicate tendencies within their first rating by moving the card on a small 5-stage scale. This second rating was supposed to reveal a more differentiated view, because often a tendency towards central values can be observed when using rating scales. The rating matrix then served as a basis for further questioning. So after completing the rating, participants were encouraged to comment on the warning modalities and were questioned about their experiences with both warning systems. Of interest were for instance which modality they liked best and the reasons for their preferences. Questions also aimed at detecting major differences between both warning systems. Other aspects during this debriefing session were ideas for optimizing the action-specific system.

Table 3

Rating matrix

\begin{tabular}{|c|c|c|c|c|c|}
\hline \multicolumn{2}{|l|}{ Rating } & Glove (action-specific system) & \multirow[t]{2}{*}{ Machine (conventional system) } & \multicolumn{2}{|r|}{ Rating } \\
\hline \multirow{5}{*}{ Very appropriate } & 1 & & & 1 & \multirow{5}{*}{ Very appropriate } \\
\hline & 2 & & & 2 & \\
\hline & 3 & & & 3 & \\
\hline & 4 & & & 4 & \\
\hline & 5 & & & 5 & \\
\hline \multirow{5}{*}{ Fairly appropriate } & 1 & & & 1 & \multirow{5}{*}{ Fairly appropriate } \\
\hline & 2 & & & 2 & \\
\hline & 3 & & & 3 & \\
\hline & 4 & & & 4 & \\
\hline & 5 & & & 5 & \\
\hline$\cdots$ & $\ldots$ & & & $\ldots$ & $\cdots$ \\
\hline
\end{tabular}

\subsection{Participants}

The study was conducted from $8-24$ August, 2011 with 42 participants ( 21 male, 21 female). Two of the participants were left-handed and one was double handed. The average age was 28.3 years (SD $=1.2$ ) and the age ranged from 19 to 61 . Almost 80 percent (33) of all participants were students, the rest (9) were employed. Six of all participants did an apprenticeship. Participants were rewarded with 10 Euro for taking part in this study.

\section{Results, discussion and future considerations}

\subsection{Result presentation}

Since the experiment has just recently been completed, only parts of the results will be reported in this paper. As mentioned before, the complete set of warning modalities was presented to the participants both with the conventional system and with the action-specific glove. Accordingly, subjects were asked to assess all modalities in both warning systems on the previously introduced rating scale. For statistical analysis the software program IBM SPSS Statistics Version 19 was used.

Figure 3 shows the mean values of both system ratings according to each modality. As pictured, the grey bars mark the mean values of the actionspecific warning system and the black bars of the conventional one. Concerning the action-specific system all modalities (apart from the visual modality which is rated at least 'moderately appropriate') were considered 'fairly appropriate'. Regarding the conventional warning system greater differences in the mean values are noticeable. In contrast to the haptic modality, which is considered as 'hardly appropriate', the combination of visual and auditory received the best rating "very appropriate". 


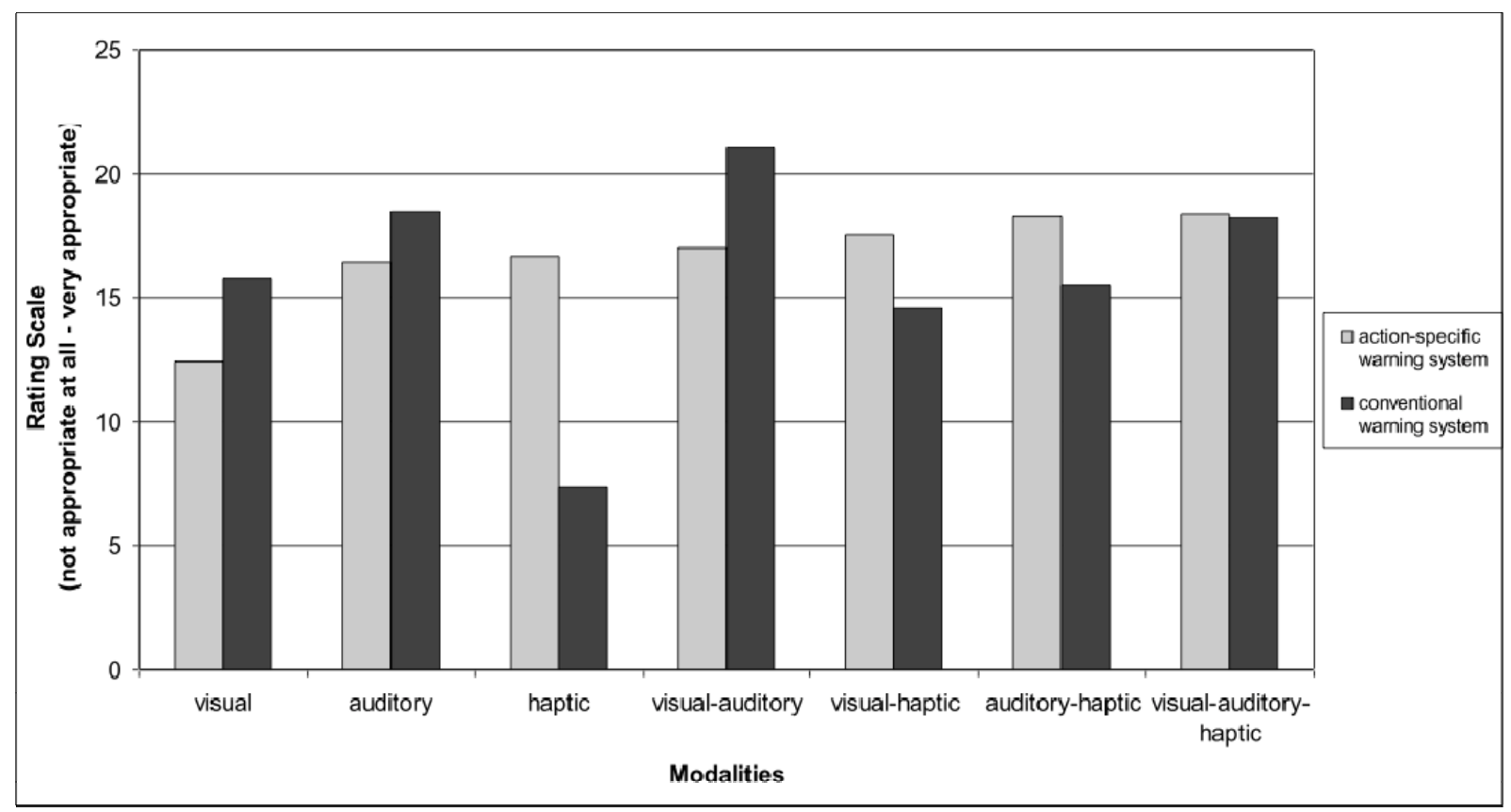

In order to analyse the ratings statistically, a twoway ANOVA with repeated measures on both factors 'system' and 'modality' was conducted. Data was corrected according to Bonferroni. As to the factor 'system' no significant main effect is reportable $(\mathrm{F}=2.57, \mathrm{df}=1, \mathrm{p}=.117)$. In contrast, the factor 'modality' has a highly significant impact $(\mathrm{F}=$ $12.42, \mathrm{df}=6, \mathrm{p}=.000)$. Furthermore, there is also a highly significant interaction effect between the two factors 'system' and 'modality' $(\mathrm{F}=28.56, \mathrm{df}=6, \mathrm{p}=$ $.000)$. According to the between-subject factor, 'test modality' (which stands for the modality the participant has experienced during the test scenario) no significant impact was found $(\mathrm{F}=.49, \mathrm{df}=6, \mathrm{p}=$ $.805)$. Individual comparisons between actionspecific and conventional system show that there are significant differences in each modality apart from the combination of all three modalities.

\subsection{Discussion}

The first statistical findings imply that the developed action-specific warning system in form of a glove was cross-modally rated as good as the conventional system, although it was only a prototype with much scope for improvements. Suggested improvements by participants of the study were for instance softer materials and wireless usage. Furthermore, it was stated that - even though it is a new way to present warnings - it could be as good or even better as a conventional system after getting familiar with it. With that, especially the visualauditory modality of the conventional system was rated as 'very appropriate' mainly because of its extensive usage in products of the everyday life. Nevertheless, participants also mentioned the risk of confusion when a warning occurs because of a lack of action-specificity and therewith not knowing the exact reason behind it: system malfunction or human mishandling.

As mentioned before, a significant effect exists according to the factor 'modality' and in the interaction between 'modality' and 'system'. That means it makes a significant difference to the user in which modality warnings are presented and which type of system is used for this purpose. A problem reported by many participants in the interview is the auditory and visual overstimulation by technical equipments within everyday life and in the working environment. Thus, especially the haptic modality on the glove was considered as a useful and practical alternative to the prevalent conventional warning systems. Furthermore, participants regarded the haptic warnings on the glove as very action-specific. Participants described it as "direct, immediate and that kind of warning, which evokes the fastest response". Thus, especially older employees will probably benefit from the earlier perceptibility and actionspecificity of those warnings because there is more 
time to select and to implement a proper action as well as a more direct link to what is going wrong.

In summary, the first results can be considered as encouragement to further develop the glove as action-specific warning system.

\subsection{Summary and future considerations}

How can multimodality be used to design usable interfaces in IPS $^{2}$ for older employees? In order to answer this opening question at first side a closer look at nowadays challenges of industrialized countries was given in the article. Secondly, the already existing theoretical concepts about human information processes, age-related effects and multimodal user interface design were considered and newly combined. As a result the concept of action-specific warnings and with that a first prototype in form of a glove with multimodal warning devices was invented. The idea behind focusing on the actionspecific component is the assumption of fewer missed warnings and a decrease in response times because of being closer to the real action and the user perception. The first experimental results of the comparison of the action-specific warning system with a conventional one lead to the conclusion that especially haptic warnings confirm that assumption. Furthermore, this invention seems to have the potential to be a purposeful alternative to conventional warning systems.

As mentioned before, the evaluation of the experimental data has only just begun and will go on in the next month. It is planned to analyze the recorded video data with regard to response times and general behavior towards the given warnings in both systems. In addition to that, a detailed qualitative content analysis of the recorded interviews will take place, in order to identify advices, ideas and suggestions for further developments of the action-specific warning system.

Additionally, further studies will be undertaken in order to test the improved versions of the glove, e.g. with softer material and in a wireless form. Moreover, different groups of users in terms of age, education level and nationality need to be tested and questioned about their experiences with action-specific concept and the prototype.

Acknowledgments. We thank the German Research Foundation (DFG, Deutsche Forschungsgemeinschaft) for funding this research within the Transregional Collaborative Research Project TRR 29 on
Industrial Product-Service Systems - dynamic interdependencies of products and services in production area.

\section{References}

[1] W.A.S. Buxton, Human skills in interface designs, in: Interaction with virtual environments, L. MacDonald and J. Vince, eds., Jon Wiley, Chichester, 1994.

[2] E. Frieling and K. Sonntag, Lehrbuch Abeitspsychologie (2 Ed.), Verlag Hans Huber, Bern, 1999.

[3] V. Hedicke, Multimodalität in Mensch-MaschineSchnittstellen, in: Mensch-Maschine-Systemtechnik - Konzepte, Modellierung, Gestaltung, Evaluation (5 Ed.), K.-P. Timpe, T. Jürgensohn and H. Kolrep, eds., Symposion Publishing GmbH, Düsseldorf, 2002, pp. 203-232.

[4] H. Meier, E. Uhlmann and D. Kortmann, Hybride Leistungsbündel - Nutzenorientiertes Produktverständnis durch interferierende Sach- und Dienstleistungen. Wt Werkstattstechnik online 95 (2005), 528-532.

[5] D.A. Norman and S. Draper, User centered system design: New perspectives on human-computer interaction, Lawrence Erlbaum Associates, Mahwah, 1986.

[6] S. Oviatt, Multimodal Interfaces, in: J. Jacko and A. Sears, eds., Handbook of Human-Computer Interaction, Lawrence Erlbaum, New Yersey, 2002.

[7] S. Oviatt, J. Bernard and G. Levow, Linguistic adaptation during error resolution with spoken and multimodal system, in: Language and Speech, Special Issue on Prosody and Conversation 41, 3-4 (1999), 415-438.

[8] J. Rasmussen, Skills, rules, and knowlege; Signals, signs, and symbols, and other distinctions in human performance models, in: IEEE transactions on systems, man, and cybernetics, SMC 13 (1983), 257-266.

[9] J. Reason, Human Error (2 Ed.), Cambridge University Press, Cambridge, 2003.

[10]B. Rohrmann, Empirische Studien zur Entwicklung von Antwortskalen für die sozialwissenschaftliche Forschung, Zeitschrift für Sozialpsychologie 9 (1978), 222-245.

[11]B. Shneiderman and C. Plaisant, Designing the User Interface - Strategies for Effective Human-Computer Interaction (5 Ed.), Addison-Wesley, Boston, 2010.

[12]H. Weißmantel and H. Biermann, Seniorengerechtes Konstruieren SENSI, Das Design seniorengerechter Geräte, VDIVerlag, Düsseldorf, 1995.

[13]C. D. Wickens, S. E. Gordon-Becker, Y. Liu and J.D. Lee, Introduction to Human Factors Engineering - International Edition (2 Ed.), Prentice Hall, New Jersey, 2003. 